\title{
Effects of exteroceptive stimuli on conditioned taste preferences induced by self-stimulation in the rat
}

\author{
AARON ETTENBERG, OWEN J. GARRETT, and PETER M. MILNER \\ McGill University, Montreal, Quebec H3A IBI, Canada
}

\begin{abstract}
Thirsty rats, permitted to drink a novel tasting solution (decaffeinated coffee) followed immediately by $15 \mathrm{~min}$ of intracranial self-stimulation (ICSS), subsequently demonstrate a preference for that novel solution. After one coffee-ICSS pairing, the preference for coffee over water in a free-choice situation was demonstrated both in the test chamber, where the pairing occurred, and in the home cage. Increases in the number of coffee-ICSS pairings, however, resulted in smaller coffee preferences in the home cage and larger preferences in the test chamber. It was suggested that as the novelty of the coffee solution decreased, the relative salience of other test chamber exteroceptive stimuli increased. After repeated coffeeICSS pairings, therefore, the rats were responding not to the presence of coffee alone, but rather to the presence of a compound stimulus of which coffee was a part. These data therefore demonstrate the importance of exteroceptive stimuli for the demonstration of conditioned taste preferences induced by self-stimulation.
\end{abstract}

In a recent paper, Ettenberg and White (1978) demonstrated that rats presented with a novel tasting substance followed by rewarding brain stimulation subsequently exhibit a preference for that novel substance. This phenomenon is, it would seem, analogous to the conditioned taste aversion (CTA) paradigm, where a novel taste followed by induced illness produces an aversion to the novel-tasting substance. Ettenberg and White (1978) interpreted their results as a demonstration of a conditioned taste preference (CTP) that occurs as a result of associations that are formed when the novel taste and the positive affective properties of the brain stimulation are paired.

Although it has been known for some time that rats will associate novel tastes with postingestional illness (Richter, 1953; Rzoska, 1953), it was Garcia and Koelling (1966) who first demonstrated that novel tastes tend to be associated most easily with particular types of aversive events. Rats associated novel tastes with sickness but not with electric shock, and audio-visual stimuli were most easily associated with shock but not with sickness. Similar results were later reported by Rozin (1969), who demonstrated that rats more readily associated the taste of a liquid with sickness than the place in which they had drunk the liquid. More recently, White, Sklar, and Amit (1977) have similarly shown that while rats will avoid

Requests for reprints should be addressed to A. Ettenberg, McGill University, Department of Psychology, 1205 McGregor Avenue, Montreal, P.Q. H3A 1B1, Canada. The critical comments of Dr. N. White and P. Pasquali are gratefully acknowledged. The author also wishes to thank C. J. Coren for assistance in testing the animals. This research was supported by National Research Council of Canada Grant A0066 to Dr. P. M. Milner. a novel-tasting substance that had previously been followed by an injection of morphine, they will nonetheless continue to run to the place where the pairings occurred. Although rats can be taught to use taste stimuli as predictors for shock and visual stimuli as predictors for illness, these associations are generally difficult to establish (Garcia, Kovner, \& Green, 1970; Rozin, 1969). It would appear, therefore, that rats do not readily associate sickness with exteroceptive stimuli, such as place, but do readily associate sickness with interoceptive stimuli such as tastes and odors.

In the CTP paradigm, a novel taste is associated with rewarding brain stimulation. Since rats will easily learn to press a lever or run to a particular place for brain stimulation (Milner, 1970; Olds, 1977), it would appear that exteroceptive stimuli can be readily associated with the stimulation. It was of interest, therefore, to determine whether, unlike CTAs, the conditioned taste preferences induced by rewarding brain stimulation could be brought under the control of exteroceptive stimuli. The present experiment was devised to examine this possibility.

\section{METHOD}

Subjects

The subjects were 24 male albino rats, weighing between 300 and $350 \mathrm{~g}$ at the time of surgery. Each rat was individually housed and provided with ad-lib access to food. Water was provided in two 100 -ml graduated Richter tubes located side by side on the front of each cage. Each rat was handled for several minutes every day for 7 days prior to surgery.

\section{Surgery}

Each rat was stereotaxically implanted with a bipolar stimulating electrode (Plastic Products Company) under $50 \mathrm{mg} / \mathrm{kg}$ 
sodium pentobarbital anaesthesia. The electrodes were aimed at the lateral hypothalamus. With the tooth bar of the stereotaxic instrument set at $3.2 \mathrm{~mm}$ above the interaural line, the electrode coordinates were: $.8 \mathrm{~mm}$ posterior to bregma; $1.5 \mathrm{~mm}$ lateral to midline; $8.5 \mathrm{~mm}$ ventral to the skull surface.

\section{Apparatus}

The self-stimulation chamber consisted of a Plexiglas cubicle (approximately $22 \times 22 \times 22 \mathrm{~cm}$ ) with a metal grid floor and one aluminum-plate wall. A lever (Lehigh Valley Electronics Model No. 121-03) protruded from the aluminum plate wall $5.5 \mathrm{~cm}$ from the side wall and at a height of $5.0 \mathrm{~cm}$ above the grid floor. Each press of the lever produced a $.5-\mathrm{sec}$ train of $60-\mathrm{Hz}$ sinewave intracranial stimulation delivered through a lead attached to a mercury slipring that was mounted atop the chamber, thereby allowing the subject freedom of movement. The apparatus was located inside a sound-attenuating box which was equipped with a $6-\mathrm{W} / 30-\mathrm{V}$ light source and a speaker that provided constant masking noise.

The home cages were approximately $35 \times 20 \times 18 \mathrm{~cm}$, had a grid floor, and were constructed of three aluminum-plate walls and one wire-mesh wall at the front of the cage. On the wiremesh wall was attached a food bin, which contained standard laboratory food pellets, and the two Richter tubes for drinking. The exteroceptive stimuli present in the test chamber were therefore very different from those present in each rat's home cage.

\section{Procedure}

The animals were allowed 10 days to recover from surgery, after which each rat was individually trained to leverpress for intracranial stimulation. Training consisted of one 30-min session, during which the current intensity for each animal was adjusted to a value that produced a steady rate of responding for the final $15 \mathrm{~min}$ of the session (current range: $15-45 \mu \mathrm{A}$ ). The animals were given no further experience with the intracranial self-stimulation until the test day.

Starting 1 week after the self-stimulation training, the amount of water drunk from the Richter tubes on the front of each cage was recorded every $24 \mathrm{~h}$ for 6 consecutive days. These amounts were used to determine the natural side preferences of each rat. On the 6th day, both tubes were removed from all cages and the rats were water-deprived for $48 \mathrm{~h}$.

Each rat was then individually placed into the self-stimulation cage, which contained a Richter tube in place of the lever. The rats were connected to the stimulator and were allowed to drink a solution of $2 \mathrm{mg} / \mathrm{ml}$ instant decaffeinated coffee in tap water for $10 \mathrm{~min}$. At the end of the drinking period the Richter tube was removed and the rats were allowed access to the lever for $15 \mathrm{~min}$ of leverpressing for intracranial stimulation. Some rats required a few trains of "priming" stimulation to ensure that they began responding immediately.

Immediately after this procedure, each rat was returned to its home cage. No liquids were available for $1 \mathrm{~h}$. Eight of the 24 animals were then given a water-coffee preference test. In their home cages, 4 of the 8 rats were presented with two Richter tubes, one containing the coffee solution and the other containing water. The remaining four animals were each given a similar test in the self-stimulation (test) chamber. In both cases, the water tubes were placed on each rat's preferred side and the coffee tubes were placed on each rat's nonpreferred side. The amounts of coffee and water consumed over the next 20 min were recorded. To ensure that each animal drank from both tubes, the tubes were presented to the animals as follows: for half the animals, the water tube was presented first. As soon as the rat sampled the water, it was removed and the coffee tube was made available. As soon as the rat sampled the coffee tube, it too was removed, and then, after approximately $10 \mathrm{sec}$, both tubes were replaced simultaneously. The remaining half of the animals were presented with the coffee tube first, then the water tube, then both, as just described.
Following the preference test, each rat that was tested in the test chamber was returned to its home cage and all liquids were removed from the eight cages for an additional deprivation period of $24 \mathrm{~h}$. Then each rat previously preference-tested in its home cage was tested for an additional $20 \mathrm{~min}$ in the test chamber, and those rats previously tested in the test chamber were tested in the home cage. Preference tests were therefore conducted in the home cage and test chamber of eight rats after one coffee/intracranial self-stimulation (ICSS) pairing.

One hour after the initial coffee-ICSS pairing, the remaining 16 rats were provided with two Richter tubes containing water for $48 \mathrm{~h}$, after which the tubes were again removed for an additional 48 -h period. All of the remaining 16 rats were then individually placed in the test chamber, where they were allowed to drink the coffee solution for $10 \mathrm{~min}$, followed once again by $15 \mathrm{~min}$ of ICSS. After this procedure, each rat was returned to its home cage with no liquids available for $1 \mathrm{~h}$. The two Richter tubes of water were then replaced on the cages for $48 \mathrm{~h}$ and then removed for another $48 \mathrm{~h}$, as previously described. This procedure continued until eight rats had experienced three coffee-ICSS pairings [a C-SS(3) group] and the final eight rats had experienced five coffee-ICSS pairings [C-SS(5) group]. After their final pairing, each rat's preference for coffee was tested both in the home cage and in the test chamber in the same manner as described for the C-SS(1) group.

Upon completion of the experiment, the rats were killed and perfused with physiological saline followed by a $10 \%$ Formalin solution. The brains were removed and fixed in $10 \%$ Formalin. The locations of the electrode tips were subsequently determined from $40-\mu$ thionin-stained frozen sections.

\section{RESULTS}

The electrode placements were located in the area of the lateral hypothalamus generally dorsolateral to the fornix, as illustrated in Figure 1. The mean coffee consumption (expressed as percent of total liquid intake) of each group during preference tests in the test chamber and in the home cage is shown in Figure 2. The paired presentation of coffee and selfstimulation resulted in a coffee preference in each group when the preference test was conducted in the test chamber where the coffee-ICSS pairings took place. When preference tests were conducted on the same animals in the home cage instead of the test chamber, the proportion of coffee consumed during the test decreased as a positive function of the number of previous coffee-ICSS pairings.

A two-way analysis of variance (with repeated measures on one factor) was computed on the arcsine transformed data. As would be expected from Figure 2, there were no reliable differences in the proportion of coffee consumed between groups. In other words, when one combines the test chamber and home cage data for each group, no statistically significant differences appear $[F(2,21)=1.07, p>.05]$. There were, however, reliable differences in coffee consumption within each group. Rats in all three groups consumed a greater proportion of their total liquid intake from the coffee tube when preference tests were conducted in the test chamber as compared to being conducted in the home cage $[\mathrm{F}(1,21)=27.91, \mathrm{p}<.001]$. The analysis also revealed a strong Within Group by 


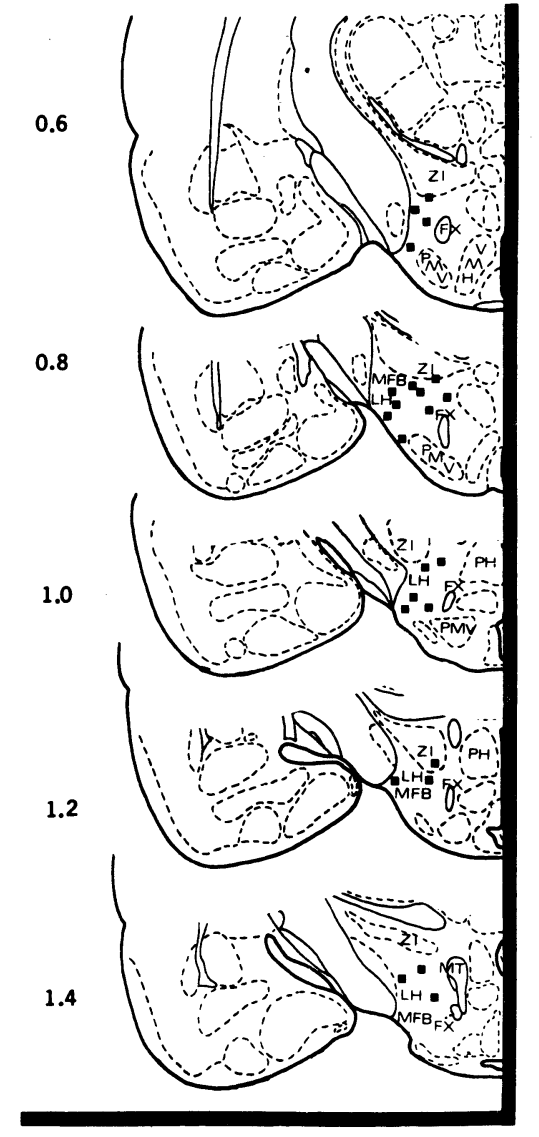

Figure 1. Electrode placements for the 24 rats used in the experiment. Numbers represent millimeters posterior to bregma. Sections are from Pellegrino and Cushman, 1967. Abbreviations: FX, fornix; LH, lateral hypothalamus; MFB, medial forebrain bundle; MT, mamillothalamic tract; PH, posterior hypothalamus; PMV, ventral premamillary nucleus; VMH, ventromedial hypothalamus; ZI, zona incerta.

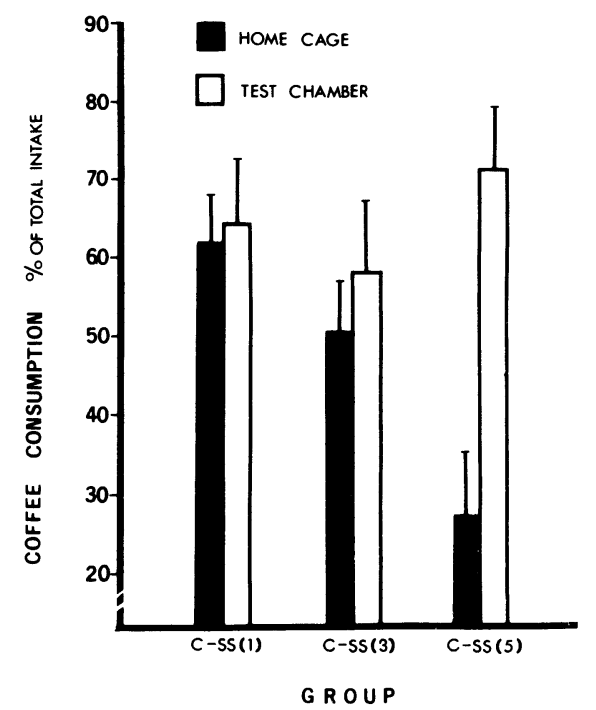

Figure 2. Mean coffee consumption ( \pm SEM) of each group during preference tests conducted in the home cage and in the test chamber. Preference tests involved 20-min coffee/water freechoice situations. Data are expressed as percent of total liquid intake. C-SS(1) = one coffee-ICSS pairing, C-SS(3) $=$ three coffee-ICSS pairings, and C-SS(5) = five coffee-ICSS pairings.
Between Group interaction, suggesting that the test chamber/home cage differences increased as a function of the number of coffee-ICSS pairings $[F(2,21)$ $=18.70, \mathrm{p}<.001]$. This is clearly illustrated in Figure 2, where the proportion of coffee consumed in the test chamber tends to increase slightly from the C-SS(1) group to the C-SS(5) group while the proportion of coffee consumed in the home cage greatly diminishes from one group to the next. [Analyses for simple effects on test chamber vs. home cage coffee consumption: $\mathrm{C}-\mathrm{SS}(1), \mathrm{t}(14)=.50, \mathrm{p}>.05 ; \mathrm{C}-\mathrm{SS}(3)$, $\mathrm{t}(14)=1.47, \mathrm{p}>.05 ; \mathrm{C}-\mathrm{SS}(5), \mathrm{t}(14)=8.05$, $\mathrm{p}<.05]$.

It should be noted that these test-chamber/homecage differences in coffee consumption did not result merely from differences in the total amounts of liquid consumed during each test situation. Table 1 shows only negligible differences in mean amounts of total liquid ingested (coffee + water) by each group during each preference test.

\section{DISCUSSION}

Rats presented with a novel tasting solution followed by a session of ICSS subsequently demonstrate a preference for that novel solution, as previously reported (Ettenberg \& White, 1978). Furthermore, as one increases the number of novel taste-ICSS pairings, the demonstration of a preference for that novel taste appears to depend more and more on exteroceptive cues-i.e., the test location. After one coffee-ICSS pairing, there was little or no difference in the performance of the C-SS(1) group in the test chamber, where it consumed $64 \%$ of its total liquid intake in coffee, compared to that in the home cage where, during the 20-min preference test, it consumed $61 \%$ of its total liquid intake in coffee. However, as the number of coffee-ICSS pairings increased, a testchamber/home-cage difference became obvious. The C-SS(3) group drank $59 \%$ of its total liquid intake in coffee when tested in the test chamber and only $50 \%$ of total intake in the home cage. This effect was most pronounced, of course, in the C-SS(5) group, where rats consumed $71 \%$ of their total liquid intake in coffee while in the test chamber and only $27 \%$ when tested in the home cage. These data might best be explained by the concepts of stimulus generalization and configural conditioning.

Mackintosh (1974) points out that subjects respond to stimuli in a generalization test to the extent that those stimuli overlap with stimuli present in the

Table 1

Mean Liquid Intake: Coffee + Water (Milliliters)

\begin{tabular}{ccc}
\hline & \multicolumn{2}{c}{ Preference Test Location } \\
Group & Home Cage & Test Chamber \\
\hline C-SS (1) & 14.6 & 13.6 \\
C-SS (3) & 13.0 & 14.3 \\
C-SS (5) & 13.8 & 12.9 \\
\hline
\end{tabular}


training situation. The slope of the generalization gradient is therefore determined by the proportion of elements common to both training and test situations. Mackintosh (1974) writes that "generalization gradients are typically orderly: responding declines in a regular manner as test stimuli depart from the training stimulus"' (p. 489). Indeed, the stimulus generalization literature has numerous demonstrations of subjects being less likely to respond when the stimulus situation during testing is markedly different from the stimulus situation present during training (e.g., Grice \& Saltz, 1950; Mednick \& Freedman, 1960; Moore, 1972). It follows, therefore, that, since the training in the present study occurred in the test chamber, one might expect a stronger conditioned response when subjects are tested in that test chamber.

While exteroceptive stimuli are generally difficult to condition in a CTA paradigm (Garcia \& Koelling, 1966; Rozin, 1969), there are good reasons to suggest that associations can be readily established between exteroceptive stimuli and rewarding brain stimulation (Olds, 1977). In the present study, the exteroceptive stimuli of the test chamber were markedly different from those present in the home cage. It is therefore conceivable that some of the exteroceptive stimuli present in addition to the coffee during pairing were also associated with the rewarding brain stimulation.

Since the coffee was probably the most salient stimulus to the thirsty rats during the first coffeeICSS pairing, one would expect the strongest association formed, after one pairing, to be that between the taste of the coffee and the rewarding properties of the ICSS. However, as the number of coffeeICSS pairings was increased, the novelty of the coffee naturally decreased, thereby increasing the relative salience of the concurrent exteroceptive stimuli. Such an increase in the relative salience of these exteroceptive stimuli should result in a strengthened association of those stimuli with the rewarding brain stimulation.

One might, for example, consider the coffee as one element in a compound stimulus which consists also of the exteroceptive stimuli of the test chamber. When viewed in this way, there are empirical reasons to believe that increasing the number of presentations of a compound stimulus followed each time by reinforcement results in a "configural" conditioning to that compound stimulus. In such instances, individual elements of the compound become less effective in producing the conditioned response. Razran (1965), for example, reviews a number of Russian studies which demonstrate that if enough compound training is given, then individual components when tested in isolation, become less effective in eliciting a CR. Other studies of configural conditioning have produced similar results (e.g., Baker, 1968; Booth \&
Hammond, 1971; Thomas, Berman, Serednesky, \& Lyons, 1968).

The present data conform neatly to this interpretation, since a stronger conditioned response (i.e., drinking coffee) would be expected when the compound stimulus is presented (i.e., coffee in the test chamber) than when one component of that stimulus is presented alone (i.e., coffee in the home cage). Furthermore, this analysis would also predict a decrease in the effectiveness of an individual component as one increases the amount of training with the compound stimulus. This, of course, is precisely what occurred in the present study where the consumption of coffee in the absence of test chamber stimuli (i.e., in the home cage) steadily decreased as a function of the number of compound (coffee in test chamber)-ICSS pairings. The present study therefore demonstrates the importance of exteroceptive stimuli for the demonstration of conditioned taste preferences induced by self-stimulation.

The CTA paradigm is generally characterized in terms of classical conditioning, where a CS (novel taste) becomes associated with a UCS (postingestional illness). Ettenberg and White (1978) have similarly proposed that the conditioned taste preference (CTP) is a parallel of the CTA model. Since, in the CTA literature, the strength of the aversion varies as a positive function of the UCS intensity (Revusky, 1968), it seemed logical to assume that the strength of the preference in the CTP model could similarly be used as an index of the rewarding value of the ICSS. Furthermore, even if one were to classify the present paradigm in terms of instrumental, as opposed to classical, conditioning, one would still predict that increases in the reward value of the ICSS should produce increases in the strength of the resulting preference. The present CTP paradigm might therefore prove to be a useful tool for assessing the value of brain stimulation reward.

\section{REFERENCES}

BAKer, T. W. Properties of compound conditioned stimuli and their components. Psychological Bulletin, 1968, 70, 611-625.

Booth, J. H., \& HAMmond, L. J. Configural conditioning: Greater fear in rats to compound than component through overtraining of compound. Journal of Experimental Psychology, 1971, 87, 255-262.

Ettenberg, A., \& White, N. Conditioned taste preferences in the rat induced by self-stimulation. Physiology \& Behavior, 1978, 21, 363-368.

Garcia, J., \& KoElling, R. A. Relation of cue to consequence in avoidance learning. Psychonomic Science, 1966, 4, 123-124.

Garcia, J., Kovner, R., \& Green, K. F. Cue properties vs. palatability of flavors in avoidance learning. Psychonomic Science, 1970, 20, 313-314.

Grice, G. R., \& SAltz, E. The generalization of an instrumental response to stimuli varying in the size dimension. Journal of Experimental Psychology, 1950, 40, 702-708.

MaCkintosh, N. J. The psychology of animal learning. London: Academic Press, 1974. 
Mednick, S. A., \& Freedman, J. L. Stimulus generalization. Psychological Bulletin, 1960, 57, 169-200.

Mrlner, P. M. Physiological psychology. New York: Holt, Rinehart \& Winston, 1970.

Moore, J. W. Stimulus control: Studies of auditory generalization in rabbits. In A. H. Black \& W. F. Prokasy (Eds.), Classical conditioning II: Current research and therapy. New York: Appleton-Century-Crofts, 1972.

OLDs, J. Drives and reinforcements: Behavioral studies of hypothalamic functions. New York: Raven Press, 1977.

Pellegrino, L. J., \& Cushman, A. J. A stereotaxic atlas of the rat brain. New York: Meredith, 1967.

RAzRAN, G. Russian physiologists' psychology and American experimental psychology. Psychological Bulletin, 1965, 63, 42-64.

Revusky, S. H. Aversion to sucrose produced by contingent $\mathrm{x}$-irradiation: Temporal and dosage parameters. Journal of Comparative and Physiological Psychology, 1968, 65, 17-22.
RICHTER, C. P. Experimentally produced behavior reactions to food poisoning in wild and domesticated rats. Annals of the New York Academy of Sciences, 1953, 56, 225-239.

Rozin, P. Central or peripheral mediation of learning with long CS-UCS intervals in the feeding system. Journal of Comparative and Physiological Psychology, 1969, 67, 421-429.

Rzoska, J. Bait shyness, a study in rat behaviour. British Journal of Animal Behaviour, 1953, 1, 128-135.

Thomas, D. R., Berman, D. L., Serednesky, G. E., \& Lyons, J. Information value and stimulus configuring as factors in conditioned reinforcement. Journal of Experimental Psychology, 1968, 76, 181-189.

White, N., SkLAR, L., \& Amit, Z. The reinforcing action of morphine and its paradoxical side effect. Psychopharmacology, 1977, 52, 63-66.

(Received for publication December 12, 1978; revision accepted February 6, 1979.) 